\title{
Abstracts from the 51st European Society of Human Genetics (ESHG) Conference, in conjunction with the European Meeting on Psychosocial Aspects of Genetics (EMPAG)
}

\author{
(c) European Society of Human Genetics 2019
}

June 16-19, 2018, Fiera Milano Congressi, Milan Italy

The ESHG conference delivered the latest findings in the field of human genetics, both basic and applied. The conference was held in conjunction with the European Meeting on the Psychosocial Aspects of Genetics, honoring the multidisciplinary and international scope of the Society. Additional information about the event may be found on the conference website: https://2018.eshg.org/

Sponsorship: Publication of this supplement was sponsored by the European Society of Human Genetics. All content was reviewed and approved by the ESHG Scientific Programme Committee, which held full responsibility for the abstract selections.

Disclosure Information: In order to help readers form their own judgments of potential bias in published abstracts, authors are asked to declare any competing financial interests.

Contributions of up to EUR 10 000.- (Ten thousand Euros, or equivalent value in kind) per year per company are considered "Modest". Contributions above EUR 10 000.- per year are considered "Significant".

Volume 27 I Supplement 1

\section{Abstracts from the $51^{\text {st }}$ European Society of Human Genetics Conference: Oral Presentations}

\section{Plenary Sessions}

PL1 Opening plenary lecture (PL1.1-PL1.2)

PL2 What's New (PL2.1-2.6, LB1-LB3)

PL3 Mendel Lecture (PL3.1)

\section{Concurrent Symposia}

S01 Prenatal Genetics-joint with EMPAG (S01.1-S01.3)

S02 DNA damage and repair in cancer (S02.1-S02.2)

S03 Genome Organization and Function (S03.2)

S04 Genetics of dizziness (S04.2-S04.3)

S05 Large-scale genetic studies in complex diseases (S05.2S05.3)

S06 Liquid biopsies in cancer (S06.3)
S07 Drug repurposing for treating genetic disorders (S07.1S07.2)

S08 Microbiome and Virome (S08.1)

S09 New Genomic Technologies (S09.1-S09.3)

S11 Epigenetics of the brain (S11.1-S11.3)

S12 Retinal diseases (S12.1-S12.3)

S13 Genome editing (S13.1, S13.3)

S14 Cellular heterogeneity in health and disease (S14.2S14.3)

S15 Understanding non-coding variants (S15.2)

S16 Human epigenome dynamics (S16.1-S16.3)

S17 ESHG-ASHG Building Bridges Debate: Germline genome editing-joint with EMPAG (S17.1S17.2, S17.4)

S18 Regulatory sequence functions and elements (S18.1S18.3)

S19 New nanotechnologies: the DNA Origami (S19.2) 


\section{Educational Sessions}

E02 Hereditary cancer (E02.1-E2.2)

E03 Resources for gene function analysis (E03.1-E3.2)

E04 Pharmacogenomics (E04.1-E4.2)

E05 Bone Density: High and Low (E05.1)

E06 Statistics in Genetic Research and Diagnostics (E06.1E06.2)

E07 Organoids (E07.1-E07.2)

E08 Congenital vasculopathies (E08.1-E08.2)

E09 Iron in the brain-joint session with the European Society of Neurology (E09.2)

E10 Genetics of infertility (E10.1)

E12 Undiagnosed disease and matchmaking initiatives (E12.2)

E13 Brain abnormalities in fetal life (E13.1-E13.2)

E15 Disorders of sexual development (E15.1-E15.2)

E16 Genetics with a Bite (E16.1-E16.2)

\section{Concurrent Sessions}

C01 Precision and Predictive Medicine (C01.1-C01.6)

C02 Syndrome updates 1 (C02.1-C02.6)

C03 Multi-omics 1 (C03.1-C03.6)

C04 Epigenetics and Gene Regulation (C04.1-C04.6)

C05 Neurological and Neuromuscular Disorders (C05.1C05.6)

C06 Internal Organs (C06.1-C06.6)

C07 NGS diagnostics (C07.1-C07.6)

C08 Population Genetics (C08.1-C08.6)

C09 Mendelian chromatin disorders (C09.1-C09.6)

C10 Best Posters 1 (P15.05A, P15.27C, P15.03C, P15.11C, P15.41A, P17.06B, P11.017A, P17.58B, P17.22B, P18.34B, P18.12D, P18.25A, P18.48D, P18.77A)

C11 Metabolic and Mitochondrial Disorder (C11.1C11.6)

C12 Skin and Bones (C12.1-C12.6)

C13 Prenatal and Reproductive Genetics (C13.1-C13.6)

C14 Cancer genetics (C14.1-C14.6)

C15 Syndrome updates 2 (C15.1-C15.6)

C16 Multi-omics 2 (C16.1-C16.6)

C17 Intellectual disability 1 (C17.1-C17.6)

C18 Cardiovascular disorders (C18.1-C18.6)

C19 Advanced sequencing technologies (C19.1-C19.6)

C20 Intellectual Disability 2 (C20.1-C20.6)

C21 Statistical Genetics (C21.1-C21.6)

C22 Best Posters 2 (P02.48C, P04.05A, P06.64D, P06.72D, P06.35C. P06.36D, P09.001A, P09.98B, P09.139C,

P12.214B, P19.24D, P20.05A, P16.40D)

C23 Sensory disorders (C23.1-C23.6)
Abstracts from the $51^{\text {st }}$ European Society of Human Genetics Conference: Posters

P01 Reproductive Genetics/Prenatal Genetics (P01.01AP01.98B)

P02 Sensory disorders (eye, ear, pain) (P02.02A-P02.60C)

P03 Internal organs \& endocrinology (lung, kidney, liver, gastrointestinal) (P03.01D-P03.46A)

P04 Skeletal, connective tissue, ectodermal and skin disorders (P04.01A-P04.91C)

P05 Cardiovascular disorders (P05.01A-P05.74B)

P06 Metabolic and mitochondrial disorders (P06.01AP06.74B)

P07 Immunology and hematopoietic system (P07.01AP07.18B)

P08 Intellectual Disability (P08.01A-P08.78B)

P09 Neurogenetic and psychiatric disorders (P09.001AP09.156D)

P10 Neuromuscular disorders (P10.01A-P10.55C)

P11 Multiple Malformation/anomalies syndromes (P11.001A -P11.103C)

P12 Cancer genetics (P12.001A-P12.216D)

P13 Basic mechanisms in molecular and cytogenetics (P13.01A-P13.33A)

P14 New diagnostic approaches, technical aspects \& quality control (P14.001A - P14.107C)

P15 Personalized/Predictive Medicine and Pharmacogenomics (P15.01A-P15.50B)

P16 Omics/Bioinformatics (P16.01A-P16.79C)

P17 Epigenetics and Gene Regulation (P18.02B-P18.79C)

P18 Genetic epidemiology/Population genetics/Statistical methodology and evolutionary genetics (P18.02BP18.79C)

P19 Genetic counselling/Education/public services (P19.01AP19.43C)

P20 Psychological/Ethical/legal issues (P20.02B-P20.16D)

\section{Abstracts from the $51^{\text {st }}$ European Society of Human Genetics Conference: E-Posters}

E-P01 Reproductive Genetics/Prenatal Genetics (E-P01.01E-P01.57)

E-P02 Sensory disorders (eye, ear, pain) (E-P02.01-EP02.17)

E-P03 Internal organs \& endocrinology (lung, kidney, liver, gastrointestinal) (E-P03.01-E-P03.41)

E-P04 Skeletal, connective tissue, ectodermal and skin disorders (E-P04.02-E-P04.18)

E-P05 Cardiovascular disorders (E-P05.01-E-P05.32)

E-P06 Metabolic and mitochondrial disorders (E-P06.01-EP06.17) 
E-P07 Immunology and hematopoietic system (E-P07.01E-P07.16)

E-P08 Intellectual Disability (E-P08.01- E-P08.37)

E-P09 Neurogenetic and psychiatric disorders (E-P09.01E-P09.45)

E-P10 Neuromuscular disorders (E-P10.01- E-P10.11)

E-P11 Multiple Malformation/anomalies syndromes (E-P11.01- E-P11.90)

E-P12 Cancer genetics (E-P12.10- E-P12.43)

E-P13 Basic mechanisms in molecular and cytogenetics (E-P13.02- E-P13.17)

E-P14 New diagnostic approaches, technical aspects \& quality control (E-P14.01- E-P14.10)

E-P15 Personalized/Predictive Medicine and Pharmacogenomics (E-P15.03- E-P15.06)

E-P16 Omics/Bioinformatics (E-P16.01- E-P16.13)

E-P17 Epigenetics and Gene Regulation (E-P17.01E-P17.02)

E-P18 Genetic epidemiology/Population genetics/Statistical methodology and evolutionary genetics (E-P18.01E-P18.16)

E-P19 Genetic counselling/Education/public services (E-P19.01- E-P19.04)
Abstracts from the 2018 European Meeting on Psychosocial Aspects of Genetics

\section{Oral Presentations}

EBPL1 Hopes and expectations on genome editing (EBPL1.1- EBPL1.4)

EBPL2 The Legal Side of Genomic Care (EBPL2.1EBPL2.4)

EPL1 Ensuring good clinical practice in whole genome sequencing (EPL1.1- EPL1.6)

EPL2 Improving communication in genetic counselling (EPL2.1- EPL2.6)

EPL3 Educating Professionals and Public (EPL3.1EPL3.6)

EPL4 What's New in Hereditary Cancer (EPL4.1- EPL4.6)

EPL5 To know or not to know (EPL5.1- EPL5.6)

EPL6 Perinatal decision-making (EPL6.1- EPL6.6)

ES1 Communication of genetic information with and within families (ES1.1)

Posters Presentations (EMP1.01.A-EMP1.79C) 